\title{
836 MURINE FECAL MICROBIOTA TRANSFER MODELS COLONIZE HUMAN MICROBES SELECTIVELY AND REVEAL TRANSCRIPTIONAL PATHWAYS ASSOCIATED WITH RESPONSE TO NEOADJUVANT CHECKPOINT INHIBITORS
}

\footnotetext{
${ }^{1}$ Fyza Shaikh*, ${ }^{1}$ Joell Gills, ${ }^{2}$ Fuad Mohammad, ${ }^{3}$ James White, ${ }^{2}$ Courtney Stevens, ${ }^{2}$ Hua Ding, ${ }^{2}$ Juan Fu, ${ }^{2} \mathrm{Ada}$ Tam, ${ }^{2}$ Richard Blosser, ${ }^{2}$ Tatianna Larman, ${ }^{2}$ Jarushka Naidoo, ${ }^{2}$ Patrick Forde, ${ }^{2}$ Sudipto Ganguly, ${ }^{2}$ Franck Housseau, ${ }^{2}$ Drew Pardoll, ${ }^{2}$ Cynthia Sears. ${ }^{1}$ Johns Hopkins School of Medicine, Baltimore, MD, USA; ${ }^{2}$ Johns Hopkins University School of Medic, Baltimore, MD, USA; ${ }^{3}$ Resphera, Baltimore, MD, USA
}

Background Human gut microbial species found to associate with clinical responses to immune checkpoint inhibitors (ICIs) are often tested in murine models using fecal microbiota transfer (FMT), wherein tumor responses in recipient mice may recapitulate human responses to ICI treatment. However, many FMT studies have reported only limited methodological description, including identification of colonizing species associated with murine outcomes, details of murine cohorts, and statistical methods. Thus, the reproducibility and robustness of ICI murine models remain uncertain.

Methods To investigate gut microbial species that impact ICI responses, we performed human to germ-free (GF) mouse FMT using pre-treatment stools from a pathologic lung cancer responder (R) and a pathologic lung cancer non-responder (NR) after neoadjuvant anti-PD-1 and anti-CTLA4 treatment, followed by implantation of the mice with syngeneic tumors and anti-PD-L1 treatment. Cohorts of GF mice varied by sex, age and syngeneic cell line implanted. To identify relevant microbes, murine tumor progressors (MT-P) and non-progressors (MT-NP) to anti-PD-L1 were classified based on tumor growth curves, 16S rRNA sequencing of human and mouse stools was performed, and data was statistically corrected for mouse characteristics using a generalized linear model. RNA sequencing was performed to assess transcriptional changes in murine tumors.

Results R-FMT mice yielded a greater anti-tumor response in combination with anti-PD-L1 treatment compared to NRFMT, although the magnitude varied depending on the mouse cell line, sex, and individual experiment. Microbiota analysis revealed a shared presence of the most highly abundant taxa between the human inocula and mice, however low abundance human taxa colonized mice more variably after FMT. Multiple Clostridium species correlated with tumor outcome in individual anti-PD-L1-treated R-FMT mice. RNAseq analysis revealed differential expression of $\mathrm{T}$ cell and NK cell-related pathways in responding tumors, irrespective of FMT source, and enrichment of these cell types were confirmed by immunohistochemistry.

Conclusions This study identifies several human intestinal microbial species that may play a role in clinical responses to ICIs and suggests attention to biological variables is needed to improve reproducibility and limit variability across experimental murine models.

Ethics Approval All studies in this abstract have been approved by Johns Hopkins University Animal Care and Use and Johns Hopkins Medicine Institutional Review Board.

http://dx.doi.org/10.1136/jitc-2021-SITC2021.836 